ISSN: 0210-7287

DOI: http://dx.doi.org/10.14201/16162019985103

\title{
FRONTERAD Y LOS NOMBRES DE LAS COSAS: \\ UN NUEVO MARCO PARA LA ACTUALIDAD \\ EN LA ERA DE LOS MEDIOS INFLACIONARIOS
}

\section{FronteraD and Los nombres de las cosas: Reframing the Present in the Age of Inflationary Media}

Jovana ZujeviC

Princeton University

jzujevic@princeton.edu

Recibido: junio de 2019; Aceptado: septiembre de 2019; Publicado: diciembre de 2019

Ref. Bibl. JOVANA ZuJEVIC. FRONTERAD Y LOS NOMBRES DE LAS COSAS:

UN NUEVO MARCO PARA LA ACTUALIDAD EN LA ERA DE LOS MEDIOS

INFLACIONARIOS. 1616: Anuario de Literatura Comparada, 9 (2019), 85-103

RESUMEN: Desde la transición a la democracia hasta la crisis económica más reciente, en España se ha consolidado la Cultura de la Transición, entendida como el sistema hegemónico que, apoyándose en los medios de comunicación mainstream, produce una narrativa coherente de la actualidad. A esta «realidad» unívoca y estable se le contrapone una configuración distinta de lo real que en los últimos años ha sido reivindicada en publicaciones independientes del periodismo narrativo, tales como la revista digital FronteraD. Este artículo argumenta que FronteraD construye un nuevo marco para tratar la actualidad mediante el uso del periodismo lento, el enfoque centrado en la vida cotidiana y un carácter transnacional. De este modo, se distancia del contenido y estilo de los medios informativos tradicionales en España. Como proyecto multimedia de cultura libre, FronteraD exige nuevas aproximaciones comparativas e interdisciplinarias que 
JOVANA ZUJEVIC

FRONTERAD Y LOS NOMBRES DE LAS COSAS: UN NUEVO MARCO PARA LA ACTUALIDAD EN LA ERA

lleven a una reconsideración de las categorías convencionales del Peninsularismo e Hispanismo.

Palabras clave: Periodismo Narrativo; Medios Digitales; Cultura Libre; Cotidianidad; Transnacionalidad.

ABSTRACT: From the transition to democracy to the more recent economic crisis, the Culture of the Transition has consolidated itself in Spain as the hegemonic system which, relying on mainstream media, produces a coherent narrative of the present. This univocal and stable "reality" is counteracted by a different configuration of the real that has emerged in recent years in independent publications of narrative journalism, such as the digital magazine FronteraD. This article argues that FronteraD reframes the present through its use of slow journalism, its focus on everyday life and its transnational character. In doing so, it distances itself from the content and style of traditional news media in Spain. As a free-culture and multimedia project, FronteraD requires new comparative and interdisciplinary approaches that lead to a reconsideration of the conventional categories of Peninsularism and Hispanism.

Key words: narrative journalism; digital media; free culture; everyday life; transnationality.

En noviembre de 2009, en plena crisis económica española, el periodista y escritor Alfonso Armada fundó la revista digital FronteraD, inspirado en The New Yorker, el baluarte del periodismo narrativo en el mundo anglosajón ${ }^{1}$. Desde entonces, la revista ha evolucionado de publicación semanal a proyecto cultural multimedia, cuya hibridez, tono literario y rebelde, así como su carácter transnacional, atraen y desconciertan a la vez tanto a los expertos en medios de comunicación como a los estudiosos de la literatura. Además de la revista, el medio de FronteraD incluye una editorial de libros impresos y electrónicos y una escuela de periodismo y pensamiento crítico.

1. Debido a la calidad de sus artículos e ilustraciones sobre cuestiones políticas, sociales y culturales, su comprobación de los hechos, su periodicidad y un público de lectores amplio y diverso, The New Yorker ha adquirido en casi un siglo de su existencia la reputación de la revista más distinguida del periodismo narrativo. Este subgénero de literatura y periodismo, que usa los elementos estéticos propios de la literatura para abordar la actualidad y la vida cotidiana, es también conocido en el mundo hispano como "periodismo literario", "articulismo literario" y "literatura periodística", por mencionar solo unos pocos términos. Teniendo esto en mente, "periodismo narrativo" y "periodismo literario» se usarán como sinónimos en el presente artículo. 
Si las noticias en los periódicos y telediarios principales son seleccionadas, enmarcadas y presentadas de tal modo que incorporan las suposiciones sobre lo que importa (Schudson 2017, 14), FronteraD desafía esas mismas suposiciones y ofrece una versión alternativa de la actualidad centrándose en los temas relacionados con la cultura, el arte, la ciencia y la tecnología y en las regiones del mundo menos tratadas en los medios dominantes. David Castillo y William Egginton denominan a estos "medios inflacionarios" debido a su capacidad para influir en nuestra percepción y comprensión de la realidad o, más bien, para "editar nuestro mundo". Este proceso de edición consiste en la construcción de una versión específica de la realidad a través de enmarcar la experiencia vivida como una narrativa o un conjunto de imágenes coherente excluyendo simultáneamente cualquier elemento que pueda perjudicar la coherencia del marco establecido (Schudson 2017, 10). Con esto en mente, me propongo demostrar en el presente artículo que FronteraD perturba la cohesión de esa narrativa de la cotidianidad, denuncia la manera en que los medios inflacionarios editan la realidad y construye un nuevo marco para comprender la actualidad.

En el contexto cultural de la España contemporánea, la fabricación de una realidad oficial e incuestionable -la cual, como veremos, FronteraD propone desmantelar- se debe a la existencia de lo que el periodista y escritor Guillem Martínez denomina la "Cultura de la Transición» (CT), que define como una cultura vertical cuyo propósito principal ha sido "crear estabilidad política y cohesión social» (Martínez 2012, 16). Varios estudiosos concuerdan en que este modelo cultural se ha consolidado como el hegemónico desde los años de la transición a la democracia hasta la crisis económica más reciente. Fernández describe la CT como totalizadora y excluyente (2014, 213), Fernández-Savater la caracteriza como consensual y desproblematizadora (2011, 1-2), mientras que Moreno-Caballud (2014, 13) señala que la CT se niega a cuestionar sus propios fundamentos políticos (el sistema de partidos) y económicos (el capitalismo). Es precisamente gracias a estos fundamentos y a sus correspondientes aparatos ideológicos de Estado (Althusser 1971) -en particular los medios de comunicaciónque la CT ha perdurado como un sistema que produce y gestiona lo real (Fernández 2014, 214). Sin embargo, gracias al crecimiento de Internet y el surgimiento del movimiento por la "cultura libre» (Lessig 2004), a esta producción de una realidad unívoca, estable y coherente, se le contraponen visiones alternativas de la actualidad y de la cotidianidad que reflejan una nueva concepción de la creación cultural como serie de procesos dinámicos, colectivos y accesibles en los que puede participar cualquiera (Moreno-Caballud 2014, 13). 
A diferencia de la CT, cuya esfera de acción son las instituciones culturales nacionales y los periódicos y programas de televisión mainstream, la horizontalidad de la cultura libre se fomenta en unos espacios más abiertos y democráticos como Internet. En efecto, la Red ha sido el entorno natural de una serie de publicaciones periódicas digitales que surgen en España en la primera década de este siglo con el fin de cuestionar el discurso oficial promovido por la CT y ofrecer una configuración distinta de lo real. A través de sus contenidos, su estilo y la disolución de la frontera entre el autor y el público, estos proyectos de cultura libre pretenden desplazar el modelo tecnocrático de la sociedad que ha llevado a la crisis actual con su insistencia en que "la política, la economía e incluso "la cultura" se consideran cuestiones de expertos» (Moreno-Caballud 2014, 15). Además, estas publicaciones cuestionan la industria cultural y periodística en España mediante su temática, un cierto espíritu activista, la transnacionalidad o el cultivo de un periodismo lento ${ }^{2}$, minucioso y creativo.

Entre estas revistas y periódicos de muy distinta naturaleza ${ }^{3}$, FronteraD se destaca como la más híbrida por su contenido y organización, siendo así capaz de construir una narrativa multifacética de lo real y un nuevo marco para la actualidad. Esto se consigue en gran medida gracias a las tres características principales del medio: 1) su estilo literario y la incorporación de elementos contraculturales en la propia presentación de FronteraD y su escuela, 2) su organización innovadora y 3) su carácter trasatlántico y transnacional. Para ilustrar mi argumento, me enfocaré en los paratextos de FronteraD, en un reparto particular de secciones con títulos creativos, en sus vínculos con The New Yorker y, por último, en una de sus bitácoras titulada Los nombres de las cosas. Este blog, escrito por la autora, periodista y filósofa Laura Ferrero ${ }^{4}$, quizá es el que mejor refleja la naturaleza híbrida y transnacional de FronteraD y su visión singular de la actualidad. En última instancia, espero mostrar a lo largo de mi análisis que este proyecto de cultura libre, concebido por un periodista español pero nacido en el espacio virtual, lleva a una reconsideración y ampliación de las categorías convencionales del peninsularismo e

2. El concepto del periodismo lento será abordado más adelante en el artículo.

3. Me refiero a las publicaciones como CTXT, El Salto (anteriormente Diagonal), Babab, Lateral, Periodismo Humano, Jot Down y Anfibia, entre otras. Aunque sin duda todas merecen ser analizadas en profundidad, en este artículo podré mencionar solamente algunas de ellas.

4. Además de este blog y varios artículos periodísticos, Ferrero ha publicado recientemente la colección de cuentos Piscinas vacias (2016) y su primera novela: Qué vas a hacer con el resto de tu vida (2017). 
hispanismo puesto que exige nuevas aproximaciones comparativas, interdisciplinarias y transnacionales.

FronteraD fue inicialmente concebida como una revista digital e independiente del periodismo narrativo y en sus nueve años de existencia ha lanzado su propia editorial, ha publicado en formato impreso dos antologías de textos e ilustraciones originalmente diseñadas para el sitio web y ha establecido la Escuela errante. A pesar de su constancia y éxito, esta revista todavía no ha recibido la atención suficiente de los críticos; quiza por ser un medio multifacético, colaborativo y electrónico y no un libro impreso firmado por un autor de renombre. Sin embargo, es esencial enfatizar que el periodismo literario ha sobrevivido a la crisis de la prensa en papel y además ha seguido desarrollándose y diversificándose como género precisamente gracias a la evolución de Internet. Por ejemplo, los reportajes y las columnas de opinión cuya cantidad de palabras, temática y estilo antes eran dictados por los editores de los periódicos impresos, se han vuelto menos estrictos y más creativos en el ciberespacio. En este sentido, las publicaciones como FronteraD abren un abanico de posibilidades para el género híbrido del periodismo narrativo, tal como señalan unos pocos críticos.

Tras investigar cómo la evolución de la red influye en el periodismo narrativo, Jorge Miguel Rodríguez y José María Albalad apuntan que FronteraD pertenece al pequeño grupo de revistas que desafían el establishment mediante su relación con el público, formato y contenidos:

Se ha eliminado al intermediario, no se amarran a la publicidad ni dependen de propietarios con afán lucrativo, no están sujetas a líneas editoriales con intereses ideológicos ni mercantiles, interactúan con los lectores [...], liberan contenidos en el ciberespacio difundidos con anterioridad en las ediciones impresas, etc. (2013, 86-87).

Eva Domínguez y J. Pérez Colomé concuerdan en que Internet ha tenido un impacto positivo en el periodismo narrativo y añaden que ha llevado al surgimiento de lo que denominan "proyectos de microperiodismo" (2012, 9) y "periodismo sin prisas" $(2012,61)$. En la entrevista que estos críticos realizaron con Alfonso Armada, el editor de FronteraD declara que el periodismo narrativo anglosajón y especialmente la revista The New Yorker han sido las fuentes de inspiración principales para su publicación, que desde el comienzo ha intentado abordar aquellos temas y partes del mundo habitualmente no discutidos en los medios más tradicionales. Armada también destaca que FronteraD pretende «estimular la inteligencia y el espíritu crítico de los lectores abrumados con la velocidad y la confusión de internet» (2013, 62). De modo parecido, Rosique-Cedillo y Barranquero-Carretero consideran que FronteraD es una de las pocas revistas que denuncian 
«el culto a la velocidad ${ }^{5}$, por lo cual se puede considerar una manifestación del "periodismo slow» que "emerge como reacción a la novedad, brevedad e instantaneidad" y aspira a producir "una información rigurosa, creativa y de calidad" (Rosique 2015, 453). De acuerdo con las características del periodismo lento, los nuevos contenidos no se publican en FronteraD diaria sino semanalmente, incorporan subgéneros periodístico-literarios de largo formato y no están restringidos por lo que se considera "actual» en el momento dado $(2015,453)$.

\section{THE NEW YORKER Y FRONTERAD: DOS «REVISTAS INTELIGENTES»}

La tendencia de FronteraD a dedicarse al periodismo sin prisas, a definir su propia actualidad y a atraer y a la vez cultivar un público inteligente y omnívoro, «una inmensa minoría» en palabras de su equipo editorial (FronteraD 2015), es lo que le acerca a la revista The New Yorker. El hecho de que Armada se inspire en la principal impulsora del periodismo narrativo en la cultura norteamericana y no en las revistas publicadas en España y Latinoamérica no es nada sorprendente en la era digital e ilustra la disolución de fronteras nacionales y culturales que según varios estudiosos de los medios digitales toma lugar en el ciberespacio. Así, Martín-Barbero señala que Internet pone en cuestión el concepto de la posición geográfica fija $(2007,10)$, mientras que Lengel y Murphy sostienen que la red da la impresión de que las fronteras entre naciones y comunidades diferentes han sido eliminadas debido a una amplia gama de posibilidades comunicativas en el ámbito digital (Lengel 2001, 187). Influenciada por la reputación global de The New Yorker, FronteraD ejemplifica la convergencia espacial que caracteriza la red y aspira a convertirse en una de las promotoras principales del periodismo literario en el mundo hispano. Es más, debido a sus contenidos diversos y cuidadosamente seleccionados, así como la ingeniosidad y creatividad que evocan el tono y el estilo de The New Yorker, FronteraD se puede considerar una "revista inteligente» $\mathrm{O}$ "smart magazine».

5. Otra revista digital semanal que se define a sí misma como una publicación de periodismo lento es CTXT, que fue fundada en enero de 2015 por un grupo de periodistas procedentes de los principales diarios europeos. CTXT, cuyo lema es «orgullosos de llegar tarde a las últimas noticias", se rebela contra la inmediatez y el exceso que caracterizan la cultura digital a través del cultivo de un periodismo de alta calidad y "con un ritmo pausado" (CTXT 2018). 
Las llamadas "revistas inteligentes» empezaron a surgir en los Estados Unidos en la última década del siglo XIX y la primera del siglo XX, un período marcado por la expansión de la industria de revistas. Debido a sus textos perspicaces y con frecuencia humorísticos, a sus ilustraciones (creadas por los artistas más talentosos del país) y al tratamiento satírico que concedían a la alta sociedad, estas revistas se diferenciaban de las principales publicaciones periodísticas desde el comienzo. Además, estaban destinadas ante todo a los lectores cultos que vivían en las zonas urbanas:

Smart magazines were written and edited for the leisured classes (although not necessarily for the very rich) -for sophisticated urbanities, for the kind of person who was well travelled, well read, well acquainted; for people who wanted to be entertained, but on an exalted plane; people wishing to sidestep the bromides and sugarcoated confections being dished out to standardized middle-class culture by the mammoth of mass-circulation magazines (Douglas 1991, 9).

Esta tendencia a atraer a la élite intelectual fue solamente un «manierismo" que desapareció con el tiempo (Douglas 1991, 1-2) y las revistas inteligentes empezaron a interesar a grandes y diversas audiencias y así ganaron tanto popularidad como éxito comercial. Fundada en febrero de 1925 por el periodista Harold Ross, The New Yorker fue una de las primeras revistas que a través de su contenido y estilo promocionaba "la ingeniosidad» como su capital simbólico (Newton 2012, 65). Ross logró esto al distanciar los contenidos, el estilo y el tono de su revista del sensacionalismo que caracterizaba tanto los medios informativos como los tabloides. En cambio, creó una publicación que se centraría en los detalles menos obvios y en las historias, aparentemente sin importancia, sobre la vida cotidiana en la metrópolis. Estas y otras características de The New Yorker, que Ross delineó en su prospecto de la revista, llegaron a ser los rasgos distintivos del periodismo literario como género en los Estados Unidos y más allá. Ross concibió una publicación que combinara el texto y la imagen; que se centrara en la vida diaria en el ambiente urbano; que contara las historias que, de otro modo, nunca serían contadas; que fuera humorística e ingeniosa; que satirizara abiertamente eventos actuales y ciertas normas sociales, y que enganchara a los lectores interesados en la actualidad que quisieran ampliar su cultura general. Con la excepción del enfoque en la 
vida neoyorkina ${ }^{6}$, todas las ideas de Ross también están puestas en práctica en mayor o menor medida en FronteraD.

Quizá el elemento más importante del prospecto de Ross, que llegaría a ser fundamental para distinguir el periodismo narrativo de los medios de comunicación dominantes y los tabloides, sea su insistencia en prestar atención a los detalles y buscar el significado de los eventos en vez de simplemente relatarlos: "As compared to the newspaper, The New Yorker will be interpretive rather than stenographic. It will print facts that it will have to go behind the scenes to get, but it will not deal in scandal for the sake of scandal nor sensation for the sake of sensation» (citado en Douglas 1991, 143). La "ingeniosidad" de The New Yorker y, por consiguiente, la de FronteraD se puede observar precisamente en su inclinación a narrar e interpretar críticamente los aspectos menos evidentes de los acontecimientos políticos, debates sociales y gustos culturales generando así "la resistencia semiótica", que según el experto en los medios de comunicación John Fiske, consiste en cuestionar y rechazar los significados dominantes y desarrollar la capacidad de pensar de manera diferente (2010, 9-10). En efecto, FronteraD desmitifica la simplicidad aparente de la vida cotidiana y permite a sus lectores descubrir la perplejidad de la actualidad. Esto se hace evidente tanto en los paratextos de la revista y su escuela de pensamiento crítico como en su organización.

\section{EN CONTRA DE LA CULTURA DE NOTICIAS Y A FAVOR DE UN RENACIMIENTO CULTURAL}

Todos los paratextos de FronteraD denuncian la manera en que los medios de comunicación dominantes, que permiten la permanencia de la CT y sus vestigios, editan la realidad. Estos paratextos exhiben en mayor o menor medida un tono subjetivo y estilo literario, una serie de referencias contraculturales y un afán de entretener y dejar perplejos a los lectores. En la sección de la revista titulada "Quiénes somos», su equipo editorial define a FronteraD como:

6. The New Yorker ha evolucionado desde entonces y aunque todavía abarca los acontecimientos y las personas de interés en la ciudad (en la sección "Goings On About Town»), también informa sobre las noticias nacionales e internacionales manteniendo su tono y estilo particulares. 
... una revista de momento solo digital, con sede en la nube, centrada en el periodismo narrativo, la crónica y el ensayo (porque duda de que muchas noticias lo sean en realidad), y que intenta explicarse el mundo y explicárselo a quien se haga preguntas: una inmensa minoría, tal vez. La actualizamos todos los jueves, aunque siempre hay algún blog que cambia cada día. Se escribe en español, pero no se define como española (FronteraD 2015).

La breve presentación de la revista y sus objetivos confirma que FronteraD es una publicación de periodismo lento, más interpretativa que informativa, que reconoce que las noticias no son la representación más adecuada de la actualidad en la era de los medios inflacionarios. Esta idea se reitera en la presentación de Escuela errante al mencionar «las noticias que lo parecen y que a menudo no lo son» y también en la descripción de la segunda antología de la revista al imaginar una red «que no sea solo la de contar mentiras y sumar pinchazos como si la cantidad fuera un valor en sí mismo" aludiendo de ese modo a la aparición y difusión de noticias falsas en Internet. Como un contrapunto a estas y a un periodismo veloz de baja calidad que únicamente fomenta "el malestar de la cultura", la escuela de FronteraD cultiva el periodismo narrativo capaz de "contar el mundo que nos rodea" (Escuela errante 2017). Mediante la promoción de unos géneros y subgéneros fronterizos como crónica, reportaje y ensayo, que combinan los rasgos de literatura y periodismo, FronteraD denuncia el principio de objetividad, que se convirtió en uno de los grandes mitos periodísticos al recibir un amplio respaldo en el mundo anglosajón tras la Primera Guerra Mundial (Allan 2010, 28). En su lugar, Escuela errante propone un conocimiento del mundo que no descarte nuestra experiencia subjetiva y que integre «los sentimientos y las razones, la percepción y los argumentos. La lógica, la retórica, y la emoción» (Escuela errante 2017).

La crítica de los aparatos ideológicos de Estado, así como de las instituciones españolas que promueven una cultura vertical y consensual, se manifiesta en la escuela de FronteraD no solamente a través de la denuncia de la cultura de noticias, sino también mediante una rebelión contra el sistema educativo. Insistiendo una vez más en darle la prioridad a la calidad sobre la cantidad, Escuela errante "No desdeña la academia, pero sí sus momias, sus repeticiones y saberes orientados cada vez más a la cuenta de resultados, a la rentabilidad» (Escuela errante 2017). Este rechazo de ciertas prácticas académicas anticuadas y diseñadas de acuerdo con las leyes del capitalismo cognitivo ${ }^{7}$ se expresa tanto en el esfuerzo de Escuela errante

7. Según MORENO-CABAllud, la combinación de «la mercantilización cultural propia del capitalismo cognitivo en su versión neoliberal» y «el individualismo elitista de la tradición 
de fomentar el desarrollo de creatividad y pensamiento crítico como en el uso de unos espacios alternativos para la enseñanza. Del mismo modo que FronteraD enfatiza el hecho de que su sede está en el ciberespacio negándose a definirse en términos nacionales, Escuela errante tampoco tiene una ubicación fija -como indica su nombre- y pretende ofrecer cursos en una serie de espacios underground: "En anfiteatros y bajo los árboles, en sótanos de librerías y en iglesias abandonadas, a bordo de una furgoneta y en el estudio de un fotógrafo, en una reserva de primates y en una galería de arte, en un aula y en un museo, en un palomar y en una carpintería" (Escuela errante 2017). Es precisamente desde estos lugares, que evocan los ámbitos de la contracultura de los sesenta y setenta, que Escuela errante pretende cultivar unos "ciudadanos del renacimiento", unos pensadores creativos y completos que puedan combatir la velocidad, la confusión y el ruido del mundo actual. A fin de lograr este objetivo, es necesario empezar por reclutar a estudiantes de las inmensas minorías de lectores de FronteraD.

El uso de oxímoros como «inmensas minorías» y "ambiciosa ingenuidad» y las paradojas como «enseñanzas inútiles, las más necesarias» en la presentación de la revista (FronteraD 2015), en sus antologías y en la escuela, reflejan la complejidad de la actualidad, llena de contradicciones, y a la vez resaltan el carácter literario del medio. Esto se manifiesta también en la escritura de la palabra "antolojía», en la cual el uso de la «j» en vez de "g" recuerda claramente a la forma de escribir del poeta español Juan Ramón Jiménez. En efecto, su nombre se menciona en la presentación de la Segundaantolojía junto con las referencias a los Beatles, Federico Fellini, Juan Goytisolo y Henry David Thoreau (Librerantes 2017), reiterando una vez más la concepción de FronteraD como una revista inteligente.

\section{Un (DES)ORDEN DIGITAL: LA ORGANIZACIÓN DE FRONTERAD}

Además de los paratextos analizados, el carácter inquisitivo e intelectual de FronteraD es también manifiesto en su particular organización. Mientras que los periódicos mainstream distribuyen sus contenidos en unas secciones previamente establecidas bajo títulos como "Nacional", "Internacional», "Economía», "Ciencia», "Tecnología", "Cultura», "Deportes", etc., que facilitan la navegación tanto de las ediciones impresas como las digitales, la

\footnotetext{
burguesa moderna» dan pie a la «cultura de Estado» que surge durante la transición española y se consolida durante la época democrática $(2014,14)$.
} 
estructura inusual de FronteraD exige un público más participativo y analítico. Consciente de que la organización de un periódico tradicional provee un marco específico para la actualidad, de acuerdo con la propuesta de Egginton y Castillo, FronteraD desafía esta "catalogación» de la cotidianidad impuesta por los medios inflacionarios e introduce una nueva manera de organizar la información. Pero este medio no cuestiona solamente la estructura de los periódicos, sino también la manera en que el motor de búsqueda principal interpreta y organiza los contenidos en el ciberespacio: "FronteraD entiende que el mundo no es susceptible de ser segmentado en ámbitos artificiales como internacional, nacional, local, economía o cultura. De ahí que hayamos optado por unas secciones que no solo a Google le cuesta entender" (FronteraD 2015). Estas palabras del equipo editorial sugieren en clave de humor que los algoritmos de Google no son suficientemente sofisticados para descifrar los contenidos de FronteraD. En efecto, en términos de estructura, esta revista se puede considerar más original que The New Yorker, cuyas diferentes secciones llevan títulos más tradicionales como "Goings On About Town", "The Talk of the Town", "Fiction", "The Critics", etc. Sin embargo, es importante recordar que, a diferencia de The New Yorker -inicialmente solo una revista en papel cuya versión electrónica empieza a publicarse unos setenta años después de su fundación-, Fronte$r a D$ fue concebida desde el principio como una revista digital. Por lo tanto, su organización creativa se debe en gran medida a su formato electrónico, conforme al argumento del filósofo David Weinberger de que todo es misceláneo en el ciberespacio. Mientras que en el mundo material se emplean los mismos principios para organizar ideas y conocimientos que para organizar gobiernos, negocios y escuelas, el mundo digital propone una forma diferente de ordenar conocimientos, alterando así nuestra percepción de lo real (Weinberger 2008, 1-7). FronteraD exhibe esta tendencia mediante sus apartados poco convencionales y títulos creativos, así como a través de las diversas maneras que ofrece para acceder a artículos o de la posibilidad de pasar de una página a otra a través de hipervínculos.

Desde la página de inicio de la revista es posible visitar cualquiera de las siguientes secciones: "Brújula", que contiene noticias sobre cultura de diversas partes del mundo; "Acordeón", una parte con reportajes periodísticos, ensayos y podcasts; "Arpa", donde se encuentran perfiles, autorretratos, postales, poesía, etc.; «Universo elegante», con artículos sobre vida digital, ciencia y tecnología; "Sociedad del espectáculo", que rinde homenaje al filósofo Guy Debord y se dedica a la crítica de arte, letras, teatro y cine; y, por último, «Mientras tanto", donde se desarrollan más de cuarenta blogs de varios periodistas, filósofos 
y escritores ${ }^{8}$. Se puede acceder a todas estas secciones desde cualquier página de la revista, mientras que las etiquetas debajo de textos ofrecen la posibilidad de desarrollar un conocimiento más amplio sobre determinados temas de especial interés para los lectores. De ese modo, FronteraD confirma la observación de Weinberger sobre las ventajas de la organización miscelánea de la esfera digital en la que, a diferencia del mundo real, las cosas se asignan a múltiples lugares a la vez $(2008,14)$. Este reparto alternativo contribuye aún más a la creación de un nuevo marco para la actualidad que toma lugar en las páginas de FronteraD y particularmente en el blog de Laura Ferrero que se analizará a continuación.

\section{UNA ACTUALIDAD LLENA DE INCERTIDUMBRE: LOS NOMBRES DE LAS COSAS DE LAURA FERRERO}

Iniciado en mayo de 2013, el blog de la periodista, filósofa y escritora Laura Ferrero se publica en la sección de bitácoras de FronteraD denominada "Mientras tanto". Su peculiar título refleja el interés de la revista y de sus blogueros en lo supuestamente ordinario, en las experiencias y banalidades cotidianas que ocurren mientras tanto. Según Moreno-Caballud, esta "revalorización de lo cotidiano, de lo ordinario" es un rasgo fundamental de los proyectos de cultura libre que pretenden deslegitimar la figura del experto como fundamento del modelo tecnocrático de sociedad (2014, 29). Es por esa razón que la sección «Mientras tanto» valora los pequeños relatos que nunca llegan a la portada de los periódicos principales, habitualmente reservadas para los grandes acontecimientos históricos o, en palabras del filósofo Maurice Blanchot (1993, 244), para "History's "Something's happening”". En efecto, las decisiones políticas cruciales, las crisis económicas y las personas famosas no figuran en el blog de Ferrero que, en cambio, contiene microrrelatos y observaciones subjetivas sobre la vida diaria $\mathrm{O}$ "Nothing happens of the everyday» (Blanchot 1993, 243).

En Los nombres de las cosas, Ferrero explora ante todo la relación entre la ficción, los medios de comunicación y la cotidianidad arrojando de

8. Otra publicación original en términos de formato es El Salto, un diario «descentralizado y horizontal» que surgió en 2017 como resultado de la integración de varios medios independientes. Uno de estos fue el periódico quincenal Diagonal, cuya organización también cuestionaba la división convencional de las noticias en segmentos como local, nacional e internacional. Un ejemplo de la creatividad de El Salto es su sección de economía y finanzas titulada «El salmón contracorriente: Economía de escalera. Finanzas de patio». 
ese modo una nueva luz sobre la actualidad. En ese sentido, la temática y el estilo de su bitácora coinciden con la visión de Alfonso Armada para FronteraD: «Se pretendía una revista de información general con temas de largo aliento, cuidadosamente editados y distanciados de la actualidad más cercana porque la realidad no se agota, ni mucho menos, en la actualidad" (citado en Rosique-Cedillo y Barranquero-Carretero 2015, 456, cursivas mías). "La actualidad» de la que Armada pretende alejarse es precisamente la macronarrativa coherente de la "realidad» editada por los medios inflacionarios, para evocar una vez más el argumento de Castillo y Egginton. Ferrero, por otro lado, replantea la noción de la actualidad y escribe pequeñas historias con temas diversos que en su conjunto crean una imagen heterogénea e inestable del mundo que nos rodea. Quizá la explicación más precisa de su rechazo a escribir sobre la versión oficial de la realidad sea la siguiente: «Hace poco una periodista me preguntó por la ausencia de realidad -entendida ésta por política y actualidad- en lo que escribía. Le contesté que la crisis, a mi entender, la llevábamos todos dentro y se llamaba incertidumbre" (Ferrero 2016). En efecto, la incertidumbre es uno de los rasgos distintivos del blog de Ferrero, cuyo título, Los nombres de las cosas, resulta ser altamente irónico, y se expresa tanto a través de las dudas existenciales del propio yo autorial como mediante una escritura híbrida en la frontera entre el periodismo y literatura. Ambas se pueden observar en la entrada "Mis líos con el periodismo".

Publicada el 13 de mayo de 2015 y compuesta de un texto y una imagen de una libreta con la inscripción "Trust me. I'm a journalist", una taza de café y un bolígrafo negro, "Mis líos con el periodismo" discute las fronteras borrosas entre el periodismo y la literatura, rechaza la noción de la objetividad periodística y a la vez hace referencia a la actualidad. Esta entrada ilustra así lo que el conocido intelectual y escritor español Francisco Umbral denominó "periodismo de arte» y describió como una mezcla de «literatura, actualidad y noticias" y también como "literatura en dosis homeopáticas» (Umbral 2001, 14)9. «Mis líos con el periodismo» refleja asimismo el carácter trasatlántico y transnacional de FronteraD, puesto que su punto de partida es la travesía efectiva del océano Atlántico que Ferrero realiza durante un

9. En su breve artículo titulado "Periodismo de arte», Umbral utiliza esta denominación por primera vez para referirse a las columnas de conocidos escritores de ficción que se publican de forma regular en los periódicos principales. UMBRAL define este subgénero de literatura y periodismo como «una cosa que se pone al servicio de la actualidad, o la crea, con todos los atributos de la información, pero con una prosa subjetiva, lo que implica también un pensamiento subjetivo (libre), que viene a donar al corazón de estraza del periódico los mejores hallazgos literarios de esta hora» $(2001,13)$. 
viaje de Barcelona a Nueva York. Cuando su avión está a punto de aterrizar, la escritora experimenta una crisis de identidad provocada por el cambio de zona horaria e intensificada por su inhabilidad para articular a qué se dedica y así poder responder a la pregunta del oficial de aduana. Mientras todavía está en el avión, "ese limbo horario» la lleva a recordar uno de sus viajes anteriores a los EE. UU., a cuestionar hasta qué punto la profesión de uno determina su identidad y a reflexionar sobre su propia (in)comprensión del periodismo:

¿Qué soy? ¿Lo que he estudiado? Filosofía. Pero lo de responder «filósofa» nunca lo he visto claro. Reproduzco palabra por palabra una conversación que tuve hace exactamente un año en un control:

-Filósofa... ¿y qué hace un filósofo? ¿Pensar?

-Sí. Bueno, no. Estudié filosofía y periodismo.

$-i Y$ ?

-Que hago de todo. Multitasking (Ferrero 2015b).

Puesto que después de la primera respuesta el oficial de aduana empieza a mirarla como si fuera una persona sospechosa, Ferrero responde a la pregunta sobre su profesión diciendo que se dedica al periodismo. Sin embargo, esta otra contestación tampoco es satisfactoria y provoca la interrogación adicional, lo cual lleva a Ferrero a compartir con sus lectores la historia de sus problemas con el periodismo. Confiesa que se atrevió a estudiar esta carrera debido a su fascinación con la escritura de Tom Wolfe, Gay Talese, Ryszard Kapuściński y otros autores conocidos por su uso de técnicas literarias y por sumergirse completamente en las historias que investigaban. Inspirada por estos escritores, Ferrero acaba siendo, irónicamente, una estudiante fracasada:

Siendo honestos no destaqué por ser una alumna especialmente brillante en la carrera. Sobre todo en las asignaturas de prácticas, es decir, en las importantes. Un dato ilustrativo: fui la primera alumna en cinco promociones que repitió las prácticas de radio. Lo peor del caso no es que repitiera, siempre hay una primera vez, ¿no? Lo peor es que era tan absolutamente negada que mi equipo de radio únicamente me dejaba redactar el parte meteorológico. Y aun así suspendí (Ferrero 2015b).

El que Ferrero revele espontánea y abiertamente los episodios más vergonzosos de sus estudios universitarios confirma la observación de Steve Himmer (2004) de que uno de los rasgos más comunes del blog como medio es precisamente «mundane, banal, sometimes embarrassing personal 
content». Entonces, no es de extrañar que Ferrero siga pensando en sus problemas con el periodismo que se han ido aumentado a lo largo de los años:

reportajes suspendidos -Laura, eso que escribes es una opinión-, crónicas suspendidas -Laura, eso que escribes es una opinión-, columnas con un 4,5-Laura, esto que escribes tendría que ser una opinión-... Me perdí un poco con todo ese debate tan trillado entre la objetividad y subjetividad. En qué quedábamos: ¿no se suponía que había que ser siempre objetivos? ¿Nadie se daba cuenta de que todo y absolutamente todo lo que escribíamos no era más que pura subjetividad? (Ferrero 2015b).

La insistencia de Ferrero en que toda escritura es intrínsecamente subjetiva, así como su disposición a compartir con los lectores los momentos más desconcertantes de su relación amor-odio con el periodismo, desafía, por un lado, el concepto de la objetividad periodística y, por otro, señala las conexiones entre blogs y diarios personales. Según Amardeep Singh, las bitácoras son textos públicos que se parecen a los diarios de la temprana modernidad y que reinventan este tipo de escritura (2008, 21). Al prestar de los diarios "rhetorics of sincerity and immediacy» y al enfatizar "originality, collective ethics, and the authorial persona", los blogs implican el renacimiento de la figura del autor (21-22). Sin embargo, en el caso de "Mis líos con el periodismo", la autora no se presenta a sí misma como una creadora transcendente ni como una experta en su campo, sino como una persona común y corriente a quien le apasionan la literatura, el periodismo y la escritura y que, a la vez, no encaja en las categorías establecidas. Aunque Ferrero considera que el principio de objetividad y la supuesta búsqueda de la verdad del periodismo convencional son discutibles, cree en lo que llama " $m i$ periodismo", es decir, un tipo de periodismo cuyos temas principales sean la literatura y la escritura:

Nunca he sido capaz de escribir nada que no sea acerca de literatura. Es el único territorio en el que me siento a salvo. Escribo sobre ficción porque ahí las reglas son conocidas por todos. Nada es verdad ni es mentira, ni pretendo que lo sea. El problema llega cuando cojo el periódico y me dirijo a la sección de economía o de política y veo que abi se pontifica con datos y porcentajes. Y abi todo es verdad. Y es mentira (Ferrero 2015b, cursivas mías).

Esta observación complementa la imagen incluida en la entrada y enfatiza la ironía de la inscripción «Trust me. I'm a journalist». Combinando los elementos del ensayo metaficcional sobre el periodismo y la entrada de diario, "Mis líos con el periodismo" nos hace dudar de los principales medios informativos, pero confiar en Laura Ferrero y los contenidos de su 
blog. Es notable que Ferrero reflexiona sobre lo que el periodismo representa para ella mientras menciona varios episodios de su vida personal -su viaje a los EE. UU., el control de pasaporte que había experimentado en el pasado y su fracaso para leer el pronóstico del tiempo en la radio-. Al mismo tiempo, hace referencia a la vida en España al mencionar las elevadas tasas de desempleo y criticar Sálvame Deluxe, un programa de televisión sobre las vidas de celebridades y políticos. De este modo, Ferrero nos hace reconsiderar la imagen de la actualidad construida por los medios informativos a través de datos supuestamente incuestionables y programas de entrevistas melodramáticos y sensacionalistas. A esta representación de la actualidad, Ferrero le contrapone una versión alternativa basada en las historias personales, las experiencias cotidianas y la literatura. Su confianza en la capacidad de la ficción y el periodismo literario para articular la actualidad centrándose no en los grandes acontecimientos sino en la vida diaria son aún más evidentes en la entrada "Los ríos y la vida".

Publicada el 17 de junio de 2015, "Los ríos y la vida" es otra micronarrativa personal en que Ferrero describe un evento significativo en su vida, la visita a Gonzalo Sobejano, reconocido crítico literario y profesor de literatura española, en su casa en Nueva York. Durante su conversación, Sobejano recuerda la novela El Jarama (1955), de Rafael Sánchez Ferlosio, lo cual lleva a Ferrero a meditar sobre el poder de la ficción para descubrir y captar esos momentos y detalles que, aparentemente ordinarios e insignificantes, constituyen nuestra vida diaria. La fotografía incluida en esta entrada, que representa un columpio de cuerda colgado del árbol sobre un río tranquilo, establece un diálogo con la novela de Sánchez Ferlosio cuya acción gira en torno del río Jarama. Ferrero cita a Sobejano en este texto, quien explica su admiración por los novelistas reconocidos de la España de la posguerra: "Creo que las novelas que escribieron todos ellos -Delibes, Cela, Laforet, Sánchez Ferlosio, etc.- eran el mejor retrato de España. Novelas que no versaban sobre nada extraordinario. Solo contaban la vida en nuestro país» (Ferrero 2015a). Aunque Ferrero admite, quizá falsamente, que se siente avergonzada por no haber leído la obra seminal El Jarama ${ }^{10}$, es notable que todavía le fascina la observación de Sobejano de que esa novela ilustra el único propósito de la literatura:

10. Independientemente de si ha leído El Jarama o no, a través de esta declaración Ferrero desafía la idea del canon literario, cuya validez se ha debatido en los círculos académicos desde el surgimiento de los estudios culturales en la década de 1960. Esto es pertinente si se toma en cuenta que la novela de Sánchez Ferlosio inicialmente recibió tanto críticas positivas como negativas y que no llegó a formar parte del canon literario de literatura peninsular hasta mucho después. 
Añade, enfadado, que muchos intelectuales de la época criticaron el libro de Ferlosio diciendo que era un peñazo y, además, muy vulgar. Se quejaban de que las conversaciones no iban a ninguna parte: eran parcas y secas. ¿Pero hacia dónde tiene que ir la literatura sino justamente a ninguna parte, a contar lo pequeño, lo de cada dia? (Ferrero 2015a).

El hecho de que Ferrero decida citar en su blog estas palabras particulares inspirada en la novela, la cual (supuestamente) no conoce, muestra su propia creencia en la existencia de lazos fuertes entre la literatura y la vida cotidiana. Mientras escucha los pensamientos de Sobejano sobre El Jarama, Ferrero concluye que el atractivo de la novela, que termina cuando una joven se ahoga en el río, reside precisamente en el hecho de que logra captar simultáneamente «una historia cotidiana» y "la vida entera». De esta manera, si "Mis líos con el periodismo" ofrece una crítica explícita del modo "imparcial» en que los periódicos, la radio y la televisión enmarcan la actualidad, "Los ríos y la vida» desafía el discurso y el estilo de los medios dominantes a través de la "resistencia evasiva»"11, es decir, al evitar toda referencia a las representaciones convencionales de la vida diaria y centrarse en la literatura.

Si los medios de comunicación principales construyen una narrativa coherente y estable de la cotidianidad y, de ese modo, dirigen nuestra percepción e interpretación de la actualidad, entonces la revista FronteraD, como proyecto de cultura libre, junto con su escuela de pensamiento crítico y el blog Los nombres de las cosas proponen una nueva versión de la actualidad re-enmarcada, polifacética y a menudo llena de incertidumbre. Esto se logra por medio de una organización innovadora del formato de la revista; de su tratamiento de los temas relacionados con la vida diaria y la cultura; de la promoción del periodismo lento y de la transnacionalidad. Todo esto implica un distanciamiento del contenido y del estilo de los medios informativos y sensacionalistas, así como de las noticias falsas. FronteraD y Laura Ferrero no solamente presentan un nuevo marco para la actualidad, sino que también pretenden cultivar unos lectores menos dóciles y más capaces de cuestionar el statu quo, lo cual es especialmente pertinente en la era de los medios inflacionarios. Al darle prioridad a la subjetividad sobre la objetividad y a las historias individuales sobre las contadas por los medios convencionales, FronteraD y Ferrero nos incitan a reflexionar sobre las fronteras, a menudo inestables, entre lo real y lo ficticio, lo vivido

11. Según Fiske, el objetivo de la resistencia evasiva, como otro tipo de oposición micropolítica contra la ideología dominante, es evitar «la captura», sea esta ideológica o física (2010, 9-10). 
y lo representado. De esta manera, nos hacen evolucionar de «ciudadanos informativos", saturados de "hechos", a "ciudadanos informados", que tienen un punto de vista propio y la habilidad de reflexionar críticamente sobre la cultura de noticias (Schudson 1995, 169). Al publicar diversos relatos de valor duradero, cuya relevancia no está vinculada al momento o el lugar en que fueron concebidos, el medio de FronteraD rechaza el culto a la velocidad, dice "más verdades, aunque más despacio" (Umbral 2001, 14) y redefine la actualidad con el fin de impulsar un nuevo renacimiento cultural.

\section{REFERENCIAS}

Allan, Stuart. (1999) 2010. News Culture. Buckingham: Maidenhead: McGraw-Hill/ Open University Press.

Althusser, Louis. 1971. "Ideology and Ideological State Apparatuses (Notes towards an Investigation)». En Lenin and Philosophy and Other Essays, traducido por Ben Brewster, 127-188. Londres: NLB.

Angulo EgEA, María. 2013. "Mirar y contar la realidad desde el periodismo narrativo». En Crónica y mirada, editado por María Angulo Egea, 7-36. Madrid: Libros del K.O.

Blanchot, Maurice. 1993. "Everyday Speech". En The Infinite Conversation, traducido por Susan Hanson, 238-245. Minneapolis: University of Minnesota Press.

Castillo, David R. y William EgGinton. 2017. Medialogies: Reading Reality in the Age of Inflationary Media. New York: Bloomsbury Academic.

CTXT. 2018. "Quiénes somos/Cuentas 2017». CTXT. Web.

Domínguez, Eva y J. Pérez Colomé. 2012. Microperiodismos: Aventuras digitales en tiempos de crisis. Barcelona: UOC.

Douglas, George H. 1991. The Smart Magazines: 50 Years of Literary Revelry and High Jinks At Vanity Fair, the New Yorker, Life, Esquire, and the Smart Set. Hamden, Conn.: Archon Books.

EL SALTO. 2018. «El salmón contracorriente». Web.

ESCUELA ERRANTE. 2017. "Presentación». Web.

FERNÁNDEZ, Álvaro. 2014. "La mirada histórica: Estrategias para abordar la cultura de la transición española». Kamchatka, n. ${ }^{\circ}$ 4: 209-232.

FERNÁNDEZ-SAVATER, Amador. 2011. «El arte de esfumarse: crisis e implosión de la cultura consensual en España». El Estado Mental, n. ${ }^{\circ} 1$.

FERrERO, Laura. 2015a. "Los ríos y la vida». FronteraD. Web.

FERrero, Laura. 2015b. "Mis líos con el periodismo». FronteraD. Web.

FERRERO, Laura. 2016. "Todos nosotros». FronteraD. Web.

FISKE, John. 2010. Understanding Popular Culture. London: Routledge.

FronTERAD. 2015. "Quiénes somos». Web.

Himmer, Steve. 2004. "The Labyrinth Unbound: Weblogs as Literature». En Into the Blogosphere: Rhetoric, Community, and Culture of Weblogs, editado por Laura 
J. Gurak, Smiljana Antonijevic, Laurie Johnson, Clancy Ratliff y Jessica Reyman. Web.

LENGEL, Laura B. y Patrick D. MurPhy. 2001. "Cultural Identity and Cyberimperialism: Computer-Mediated Explorations of Ethnicity, Nations, and Citizenship". En Cyberimperialism: Global Relations in the New Electronic Frontier, editado por Bosah L. Ebo, 187-204. Westport, Conn.: Praeger.

Lessig, Lawrence. 2004. Free Culture: How Big Media Uses Technology and the Law to Lock Down Culture and Control Creativity. New York: Penguin Press.

LIBRERANTES. 2017. "Segundaantolojía", Librerantes. Web.

MARTíN-BARBERO, Jesús. 2007. «Latin American Cyberliterature: From the Lettered City to the Creativity of its Citizens". Prefacio a Latin American Cyberculture and Cyberliterature, editado por Claire Taylor y Thea Pitman, xi-xv. Liverpool: Liverpool University Press.

MARTíNEZ, Guillem. 2012. "El concepto CT». En CT o la Cultura de la Transición: Crítica a 35 años de cultura española, editado por Guillem Martínez, 11-23. Barcelona: Debolsillo.

Moreno-Caballud, Luis. 2014. "Cuando cualquiera escribe. Procesos democratizadores de la cultura escrita en la crisis de la Cultura de la Transición española». Journal of Spanish Cultural Studies 15 (1-2): 13-36.

NEWTON, Leslie. 2012. "Picturing Smartness: Cartoons in the New Yorker, Vanity Fair, and Esquire in the Age of Cultural Celebrities». The Journal of Modern Periodical Studies 3 (1): 64-92.

Rodríguez Rodríguez, Jorge Miguel y José María Albalad Aiguabella. 2013. «El periodismo narrativo en la era de internet: las miradas de Orsai, Panenka, Anfibia, FronteraD y Jot Down». En Crónica y mirada, editado por María Angulo Egea, 85-121. Madrid: Libros del K.O.

Rosique-Cedillo, Gloria y Alejandro BARRANQUero-CARRETERO. 2015. "Periodismo lento (slow journalism) en la era de la inmediatez. Experiencias en Iberoamérica”. El Profesional de la Información 24 (4): 451-462.

SCHUDSOn, Michael. 1995. The Power of News. Cambridge, Mass: Harvard University Press.

SingH, Amardeep. 2008. "Anonymity, Authorship, and Blogger Ethics». Symploke 16 (1-2): 21-35.

Umbral, Francisco. 2001. "Periodismo de arte». En Los placeres y los días, 13-14. Madrid: Fondo de Cultura Económica.

Weinberger, David. 2008. Everything is Miscellaneous: The Power of the New Digital Disorder. Nueva York: Holt. 
